\title{
Polymer Delivery of Hydroxycamptothecin against C6 Glioma
}

\author{
Jing Hu, Huafei Tang, Songqing Liu* \\ Department of Pharmacy, Southwest Hospital, Third Military Medical University, Chongqing, China \\ Email: songqingliu@hotmail.com
}

Received 7 July 2014; revised 1 August 2014; accepted 28 August 2014

Copyright (C) 2014 by authors and Scientific Research Publishing Inc.

This work is licensed under the Creative Commons Attribution International License (CC BY).

http://creativecommons.org/licenses/by/4.0/

(c) (;) Open Access

\section{Abstract}

Hydroxycamptothecin is a potent antineoplastic agent that has shown efficacy against multiple tumor lines in vitro. This is the first study to investigate the release, distribution, and efficacy of hydroxycamptothecin which was incorporated into the biodegradable polymer Polylactic Acid (PLA), and implant into brain directly. In vitro release curve generated showed that a large initial release occurred over the first three days and was followed by a steady, but considerably slower rate of release over the next 25 days. After implanting the discs into 40 male SD rats, the animals were followed up to 28 days, where the concentration in brain tissue was far higher than that in peripheral blood at the each of the eight time-points evaluated, and it was also within the therapeutic range for $\mathrm{C} 6$ cells tested in vitro. The in vivo efficacy of the discs was evaluated with rats inoculated intracranially with $\mathrm{C6}$ glioma and treated with hydroxycamptothecin polymer compared to intravenous as well as intratumoral injections; the median survival is $21.1,13.9,14.9$ days, respectively. Given these data, we conclude that the biodegradable polymer PLA releases hydroxycamptothecin, producing tumoricidal levels in brain tissues and prolonging survival in a rat glioma model.

\section{Keywords}

Hydroxycamptothecin Polylactic Acid (PLA) C6 Glioma Sustained Release

\section{Introduction}

Brain tumors result in many deaths every year. The conventional treatment for glioma has been surgical debulking of the accessible tumor from the patient's brain while limiting tumor relapse through post-surgical chemo-

${ }^{*}$ Corresponding author. 
therapy and radiotherapy over a period of time. Until recently, chemotherapy has been administered via intravenous injection with limited clinical effectiveness due to restricted transport across the blood brain barrier (BBB). High systemic concentrations necessary to cross the BBB often lead to several side effects. Consequently, the use of surgically implanted local release systems made of biodegradable polymers, which deliver drugs over extended periods of time, has good potential in the treatment of glioma [1]-[3].

Hydroxycamptothecin has been extracted and synthesized by Chinese scientists from Camptotheca accuminata, which is a native plant in China [4]. It is a cytotoxic alkaloid with strong antitumor activity against a wide variety of experimental tumors [5] [6], for which the intracellular target is eukaryotic DNA topoisomerase I. To effect cytotoxicity, hydroxycamptothecin must exist as a closed-ring lactone $(\mathrm{pH}<6.5)$. As such, when the lactone ring is opened by hydrolysis $(\mathrm{pH}>7.4)$, the drug is inactivated. Hydroxycamptothecin sodium is the form most typically used in clinical practice due to its solubility. However, this open conformation leads to unstable product quality and decreased efficacy. Moreover, its significant toxicity to bone marrow and hematologic systems is not permissible. Thus, these limitations largely restrict its clinical usage.

To maximize antitumor effects and minimize systemic toxicity, we incorporated hydroxycamptothecin into a Food and Drug Administration (FDA)-approved, implantable, biodegradable polymer matrix, polylactic acid (PLA), that locally delivers the drug to the tumor bed, not only bypassing the BBB and directly acting on brain tissue but also extending the functional time of the drug action with increased concentration resulting from sustained release.

\section{Materials and Methods}

\subsection{Materials}

The polymer used in the fabrication of discs was PLA (mol. wt. 20000) and was purchased from Shandong Medical Equipment Institute (SD, China). Hydroxycamptothecin was purchased from a biotechnology limited company of Guanghan (SC, China). The C6 glioma cell line was a generous gift from the Pathology Department of Southwest Hospital (CQ, China). Cells were grown in RMPI-1640 (Sigma) medium enriched with 10\% fetal bovine serum (Sigma) in a humidified atmosphere of $5 \% \mathrm{CO}_{2} / 37^{\circ} \mathrm{C}$.

\subsection{Polymer Disc Preparation}

PLA polymer containing hydroxycamptothecin was fabricated according to direct compression [7]. Combining hydroxycamptothecin with PLA produced a $20 \%$ hydroxycamptothecin polymer mixture by weight, where the mixture was sieved through 80 meshes to produce a well-mixed powder. Hydroxycamptothecin polymer discs (12 mg, final weight) were prepared by compression molding. For this, 12.0 - $13.0 \mathrm{mg}$ of hydroxycamptothecin polymer powder was prepared using a stainless steel mold (internal diameter, $3.0 \mathrm{~mm}$ ) under pressure at $250 \mathrm{~N}$ as described by Leong et al. [8] [9].

\subsection{Assays of Hydroxycamptothecin Release from Polymers}

Measuring the release of hydroxycamptothecin involved placing a hydroxycamptothecin-loaded polymer disc into a vial with $10 \mathrm{ml}$ of $0.1 \mathrm{M}$ phosphate-buffered saline containing $1 \%$ sodium dodecyl sulfonate (SDS), $\mathrm{pH}$ 6.5 , and incubating at $37^{\circ} \mathrm{C}[10]$. The solution was removed at various time points and replaced with fresh buffer, and the recovered solution was assayed for the presence of hydroxycamptothecin by high-pressure liquid chromatography (HPLC). These analyses were performed with a Waters Alliance 2690 HPLC system (consisting of a 474 Fluorescence detector, Millennium chromatography workstation), equipped with a $4.6 \times 250 \mathrm{~mm} \mathrm{C}_{18}$ Waters column (KROMASIL, particle size $10 \mu \mathrm{m}$ ). The flow rate was $1 \mathrm{ml} / \mathrm{min}$, where the mobile phase consisted of methanol/water (55:45), and $\mathrm{Ex}=363 \mathrm{~nm}, \mathrm{Em}=550 \mathrm{~nm}$ [11]. Under these conditions, the retention time of hydroxycamptothecin was $6.90 \mathrm{~min}$. Constructing a standard curve required plotting the peak area against concentration.

\subsection{Animals}

Male SD 90 rats weighing 200 - 250 g were obtained from the Experimental Animal Sector of the Third Military Medical University (CQ, China). All rats were housed in standard animal facilities and were given free access to 
water and food.

\subsection{Anesthesia}

Rats were anesthetized with an i.p. injection of 2 - $4 \mathrm{ml} / \mathrm{kg}$ of a stock solution containing ketamine hydrochloride $(25 \mathrm{mg} / \mathrm{ml})$, xylazine $(2.5 \mathrm{mg} / \mathrm{ml})$, and $14.25 \%$ ethyl alcohol in normal saline.

\subsection{Hydroxycamptothecin Concentration Comparison between Intra-Cerebrum and Peripheral Blood}

Polymer discs were prepared as described above and were sterilized by irradiation with $20 \mathrm{kGy} \mathrm{CO}_{60}$. The procedure for polymer disc implantation in the rat is described elsewhere [12]. Briefly, anesthetized rats had their heads shaved and were prepared aseptically. Exposing the dura required a midline incision and a 3-mm burr hole drilled through the skull $5 \mathrm{~mm}$ posterior and $3 \mathrm{~mm}$ lateral to the bregma. Incising the dura with a microsurgical knife exposed the brain parenchyma and allowed the insertion of a polymer disc. The incision was irrigated and closed with surgical clips.

At 1, 3, 7, 10, 13, 17, 21 and 28 days after implantation, groups of five rats each were sacrificed. The polymer disc was removed, and the brain tissue was then processed. Continuous normal saline irrigation was used to clean and then to weigh. The brain was then homogenized with normal saline at the ratio of $5 \mathrm{ml} / \mathrm{g}$ and was centrifuged (13,000 rpm, $5 \mathrm{~min})$. At the same time points, blood samples $(0.5 \mathrm{~mL})$ were collected from the tail vein before sacrificing, followed by centrifugation ( $4000 \mathrm{rpm}, 5 \mathrm{~min}$ ). HPLC was then used to measure the amount of hydroxycamptothecin in the brain and plasma samples. Equipped with a $4.6 \times 250 \mathrm{~mm} \mathrm{C}_{18}$ Waters column (Diamonsil, particle size $10 \mu \mathrm{m}$ ), the mobile phase consisted of acetonitrile $/ 0.1 \%$ triethylamine $(25 / 75, \mathrm{v} / \mathrm{v}, \mathrm{pH}=$ $3, \mathrm{H}_{3} \mathrm{PO}_{4}$ ).

\section{Efficacy Studies}

\subsection{Animal Model}

The C6 glioma model was used as a brain tumor model here [13]. C6 glioma cells were harvested by trypsinization, pelleted by centrifugation, and resuspended for intracranial implant, and SD rats were placed in a stereotactic frame (SN-1, Naishge Scientific instrument Lab, Japan). After a midline scalp incision of the shaved skin, local disinfection and identification of the sagittal sutura and the Bregma (the crossing point with the coronal sutura) were performed, and a burr hole of $1 \mathrm{~mm}$ in diameter was drilled $3 \mathrm{~mm}$ lateral and $1 \mathrm{~mm}$ behind this position. Through this burr hole, a 25-gauge needle was inserted vertically to a depth of $5 \mathrm{~mm}$. The cell suspension $\left(1 \times 10^{9}\right.$ cells in $\left.10 \mu \mathrm{l}\right)$ was injected within 5 min using a microinjector. The needle was slowly removed to avoid an undertow following removal of the injected suspension through the insertion canal, the burr hole was sealed with bone wax, and the skin was closed using surgical sutures.

\subsection{Animal Group}

C6 glioma formation was achieved after 10 days as determined by MRI, and rats were randomized into five groups of 10 animals each. After re-anesthetizing the rats, the initial incisions were reopened under sterile conditions, where control group animals received no further treatment, and the blank implant group received PLA discs containing no drug. There were three treatment groups, as follows: an implanted treatment group where each animal was implanted with a $12 \mathrm{mg}$ PLA disc containing 20\% hydroxycamptothecin; an I.V. group infused with $2 \mathrm{mg} / 1 \mathrm{~kg}$ hydroxycamptothecin; and an intratumoral injection group that received $50 \mu \mathrm{l}(2.4 \mathrm{mg})$ of the hydroxycamptothecin solution injected at a depth of $3.5 \mathrm{~mm}$ below the brain surface.

Rats in each group were evaluated on a daily basis for weight, signs of consciousness, movement defects, or an inability to feed or groom themselves. As such, survival time was the key parameter evaluated.

\subsection{Statistical Analysis}

Survival was plotted on a Kaplan-Meier survival curve, and statistical significance was determined by a nonparametric Kruskal-Wallis analysis of variance followed by a nonparametric Wilcoxon Rank SumTest. Values 
were considered significant for $\mathrm{P}<0.05$.

\section{Results}

\subsection{In Vitro Drug Release}

Release of hydroxycamptothecin from biodegradable PLA polymer is shown as a cumulative percentage of the total drug loaded into the polymer in Figure 1. For 20\% hydroxycamptothecin loading, a large initial release occurred over the first 1 - 3 days. The initial, rapid release was followed by a steady, but considerably slower rate of release over the next 25 days. At the end of the experiment, the discs released $80 \%$ of their total loading dose (2.4 mg of hydroxycamptothecin) into the buffer. The initial release was as high as $32 \%$, which resulted from drug dissolution at the surface and diffusion from internal attributes due to osmotic pressure. After the initial release, discs demonstrated a steady and slower release for the remainder of the experiment according to degradation of PLA and drug diffusion internally, for which the release rate was maintained for approximately one month.

\subsection{Concentration Comparison}

In order to evaluate the systemic toxicity of the polymer discs, we detected the concentration of hydroxycamptothecin in brain tissue and in peripheral blood. As such, we chose eight time points during the course of the release of polymer discs implanted into the rats' brains. As Figure 2 shows, the concentration in the brain was much higher than in the peripheral blood at each of the eight time points evaluated. The concentration in the brain was greater than $130 \mu \mathrm{g} / \mathrm{ml}$, and the maximum concentration achieved was $183.16 \mu \mathrm{g} / \mathrm{ml}$, which was far higher than the $\mathrm{IC}_{50}$ (per $\mathrm{C} 6$ glioma cells following hydroxycamptothecin treatment in vitro). In contrast, the concentration in peripheral blood was only about $150 \mathrm{ng} / \mathrm{ml}$, which was far lower than in the brain. This result suggests that polymer discs directly implanted into the brain enable a high concentration of the drug to be maintained directly in the brain for approximately one month, whereas the concentration in the peripheral blood was lower, reducing systemic toxicity while improving inhibition of glioma cells.

\subsection{Efficacy Studies}

We evaluated the efficacy of the hydroxycamptothecin polymer discs in a C6 glioma model. We studied the efficacy of the polymer discs against not only a no treatment group (control) but also a group treated with intravenous injection ( $2 \mathrm{mg} / \mathrm{kg}$ ) and a group treated with intratumoral injection (50 $\mu 1,2.4 \mathrm{mg})$. After about 10 days, the C6 glioma was formated, as shown in Figure 3. In the course of the formation, animal weights' were gradually reduced, with all animals demonstrating neurocognitive symptoms to different degrees, reduced motor activity, and an inability to feed and groom themselves.

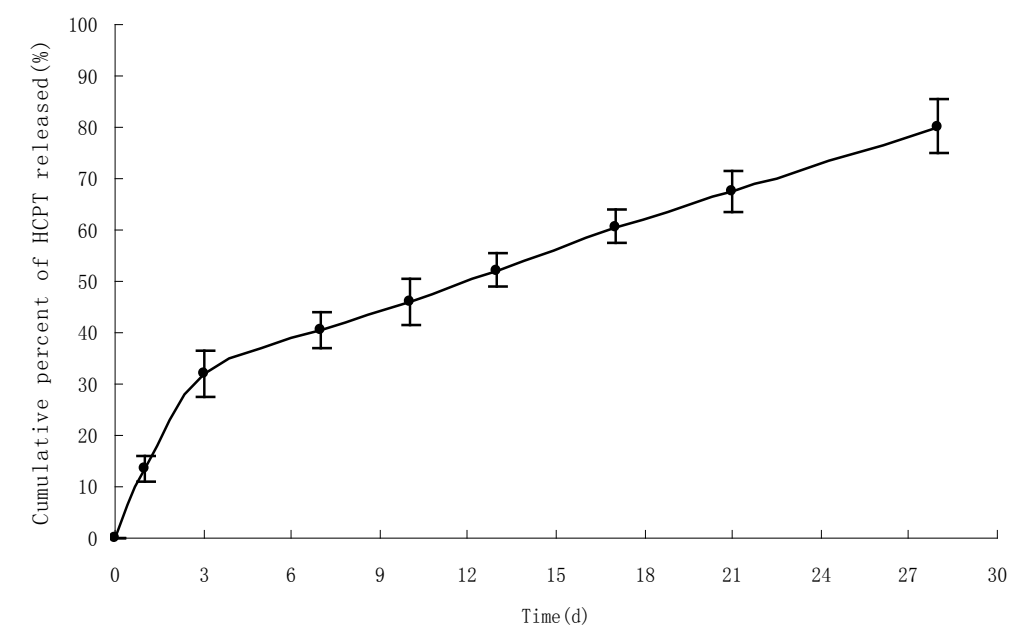

Figure 1. Cumulative percentage of hydroxycamptothecin released from polymer discs $(n=3)$. 


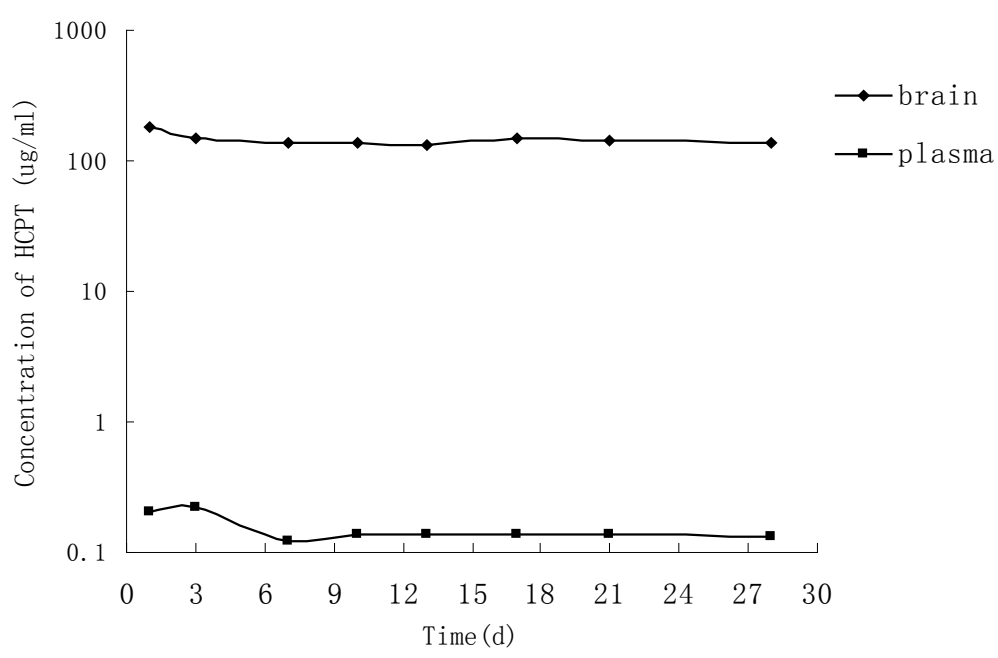

Figure 2. The concentration of hydroxycamptothecin in the brain and plasma $(\mathrm{n}=5)$.

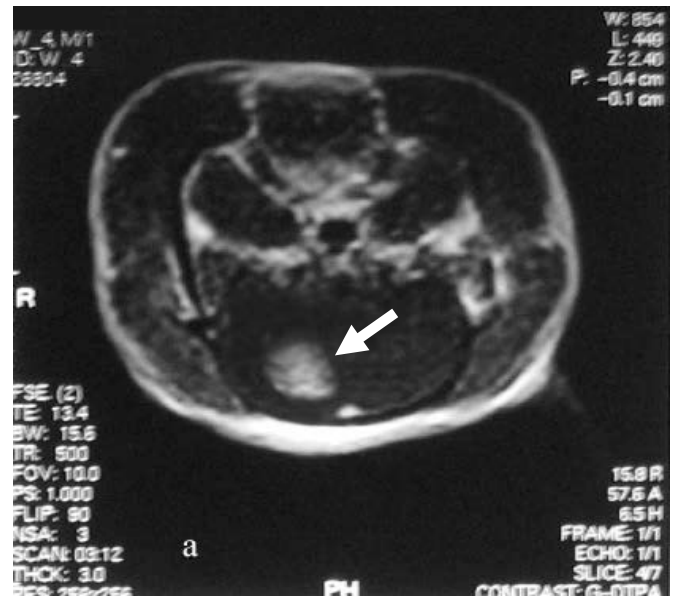

(a)

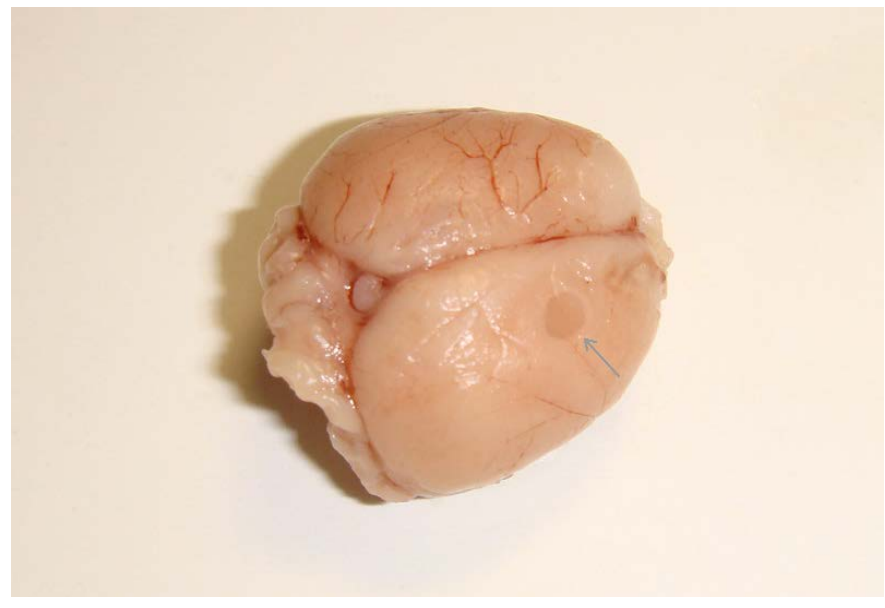

(b)

Figure 3. The formation of C6 glioma in rat brain. (a) The MRI of formed C6 glioma in rat brain; (b) The sample of formed C6 glioma in rat brain.

For the group treated with hydroxycamptothecin polymer discs, the tumor size decreased to some extent, as shown in Figure 4. The survival rates were better, but the other two treatment groups did not show improvement. Kaplan-Meier survival curves for this are shown in Figure 5. The blank group was similar to the control group, and the I.V. group did not significantly extend survival compared to the control group. However, the rats that were treated with intratumoral injection extended survival to some extent, although not significantly compared to the control group, such that the P values of the I.V. group and intratumoral injection group were 0.125 and 0.053, respectively. However, the $20 \%$ hydroxycamptothecin polymer discs significantly extended survival compared to the control group $(\mathrm{P}<0.001)$, and significantly compared to both the I.V. and intratumoral injection groups ( $\mathrm{P}<0.001$ for both). The median survival times for the $20 \%$ hydroxycamptothecin polymer, I.V., and intratumoral injection groups were 21.1, 13.9, and 14.9 days, respectively. The median survival for the control group was 12.9 days, and the blank PLA group was 13.2 days, as shown in Table 1.

\section{Discussion}

Delivering chemotherapeutic agents intracranially bypasses the blood-brain barrier and allows the drug to be delivered directly to the tumor bed. The blood-brain barrier and severe systemic toxicity have restricted potentially effective systemic chemotherapy treatments for malignant glioma. A myriad of chemotherapeutic agents 


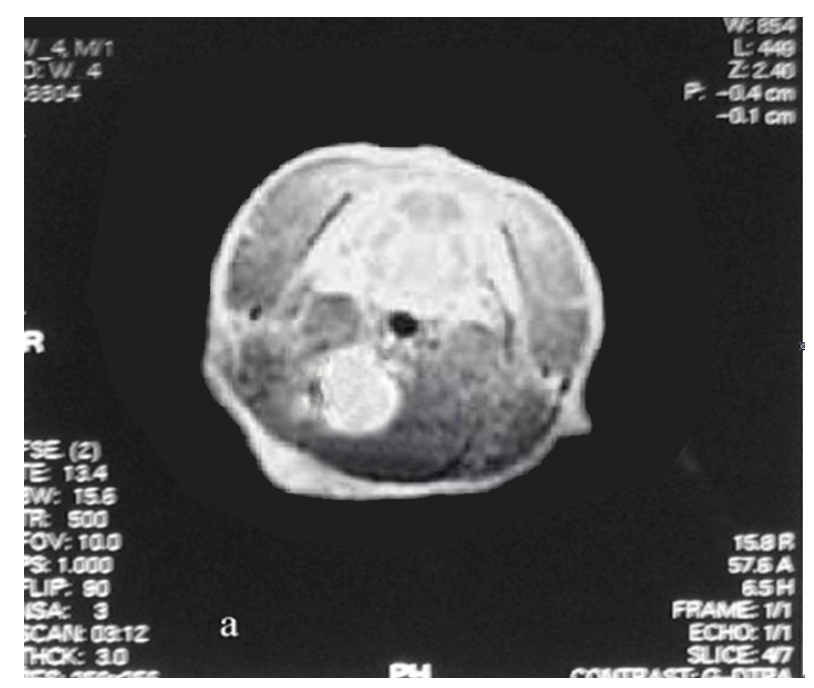

(a)

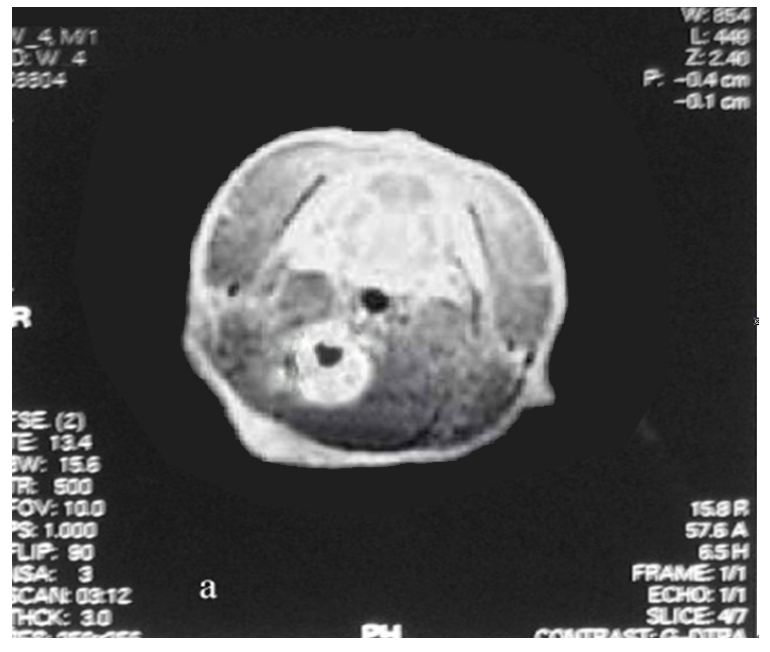

(b)

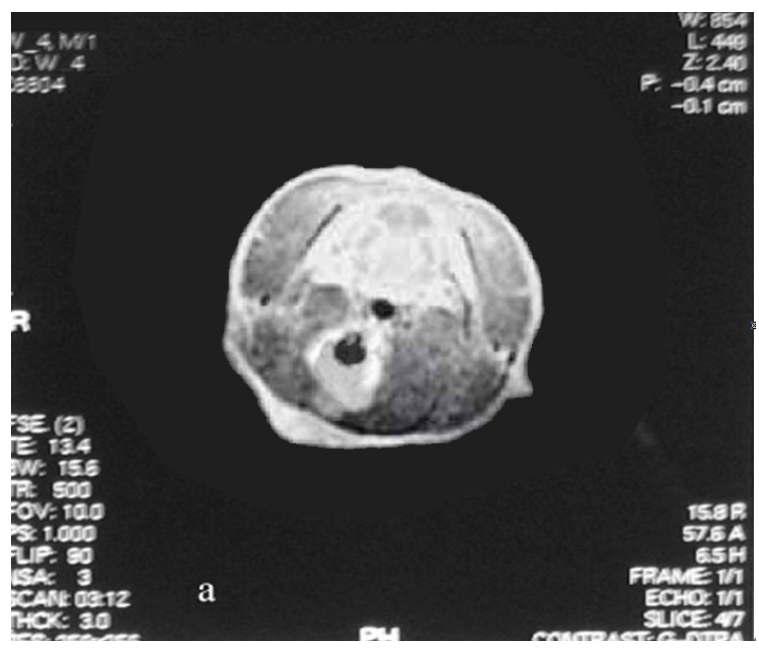

(c)

Figure 4. MRI scans after implantation of hydroxycamptothecin polymer discs. (a) Preoperatively; (b) 10 days after implantation; (c) 15 days after implantation.

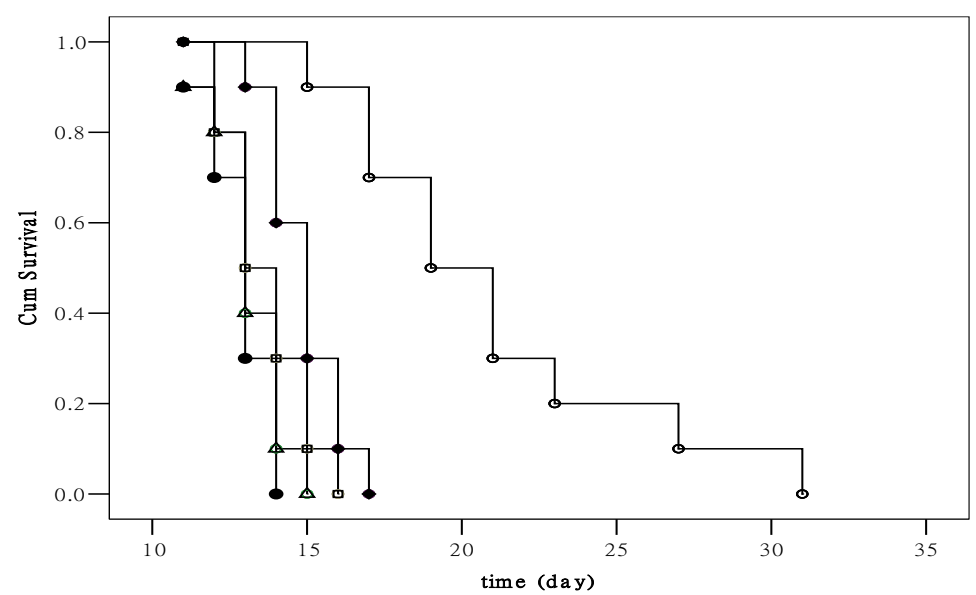

Figure 5. Survival analysis of the treated groups $(\mathrm{n}=10)$. $\square$ I.V.; $\diamond$ intratumoral; o implant; $\bullet$ control; $\Delta$ plasma. 
Table 1. The comparison of survival time between groups $(n=10)$.

\begin{tabular}{|c|c|c|c|c|}
\hline Group & Median survival time (d) & P value $^{\mathrm{a}}$ & P value ${ }^{\mathrm{b}}$ & P value $^{c}$ \\
\hline Control & 12.9 & & & \\
\hline Blank & 13.2 & 0.438 & & \\
\hline I.V. & 13.9 & 0.125 & & \\
\hline Intratumoral injection & 14.9 & 0.053 & & \\
\hline Implant intracal & 21.1 & $<0.001$ & $<0.001$ & $<0.001$ \\
\hline
\end{tabular}

${ }^{\mathrm{a}}$ Results of nonparametric Wilcoxon Rank Sum Test comparing treatment groups to controls; ${ }^{\mathrm{b}}$ Results of nonparametric Wilcoxon Rank Sum Test comparing Implant Intracal groups to the I.V. group; ' Results of nonparametric Wilcoxon Rank Sum Test comparing Implant Intracal groups to the Intratumoral injection group.

have shown promising in vitro results against malignant glioma, only to fail in clinical trials, because they cannot cross the blood-brain barrier at cytotoxic concentrations or because of unacceptable systemic toxicity.

Hydroxycamptothecin has been previously shown to have significant antineoplastic actions in vitro [14], but its clinical use as a systemic agent has been limited by severe toxicity and weak water solubility [15]. For this reason, we chose to study hydroxycamptothecin in our local chemotherapy delivery model. Other factors making hydroxycamptothecin an attractive agent include its unique mechanism of action (allowing for potential synergy and multiple drug therapy) [14].

In this study, we investigated the release, concentration comparison, and efficacy of hydroxycamptothecin delivered intracranially from a biodegradable polymer. The polymer PLA has been well studied and applied, and its biocompatibility has been shown to be favorable [16]. As such, it has been used in implantations for the purposes of delivering multifold drugs, while having a good slow-release effect [17] [18].

Polymer degradation proceeds with zero-order kinetics; therefore, it was predicted that the amount of drug released would be constant with respect to time. Instead, it was found that in vitro drug release from the polymer matrix occurs with an initial, rapid-release phase followed by a slower and more constant release [19]. The in vitro data presented here also show this biphasic pattern. The initial, rapid release phase is likely related to the presence of hydroxycamptothecin on the surface of the polymer and weak binding to the polymer. At this stage, the release of the drug increases drastically and is related to the drug loaded into the polymer. After the initial burst, the observed kinetics achieve a stable release stage, which is functionally the most important stage of release. The external release medium diffuses internally, while causing the drug to diffuse into the external phase. Simultaneously, the PLA degraded gradually, promoting diffusion of the drug. Therefore, the stable release process is co-controlled by diffusion and degradation.

The purpose of preparing a polymer implant is to enable the concentration of hydroxycamptothecin to increase in brain tissue, but to remain lower in peripheral blood, so as to maximize efficacy and minimize systemic toxicity. After implanting hydroxycamptothecin polymer discs intracranially, we compared the concentration in brain tissues and in peripheral blood. The results show that the concentration in the brain is far higher than that in peripheral blood. Just as we expected, during the course of release, the polymer discs enable the concentration of the drug to maintain therapeutic levels for a month in brain tissue, while remaining lower in other tissues. Therefore, the hydroxycamptothecin polymer discs implanted intracranially can achieve better efficacy with reduced toxicity.

Our results indicate that $20 \%$ hydroxycamptothecin polymer discs significantly prolonged survival in a rat glioma model. Inversely, the survival curves for the I.V. group and intratumoral injection group were no different from the control group. The liposolubility of hydroxycamptothecin is weak, making it difficult to pass the blood-brain barrier. Thus, I.V. treatment was unable to achieve a drug concentration representative of effective therapeutic levels in the brain. Treatment with intratumoral injection enabled the drug to act on glioma cells directly, but this approach is limited to a single dose. Moreover, the half-life of hydroxycamptothecin is short, such that the concentration cannot be maintained effectively after initial injection, while repeated injections could cause serious brain injury. The hydroxycamptothecin polymer discs implanted into the intracalvarium not only enable hydroxycamptothecin function at the target site directly but also maintain the effective therapeutic concentration for approximately one month due to its characteristic slow-release, extending the functional time of the drug, while inhibiting the tumor cell effectively. Even though the pharmacokinetics of the in vivo release 
are undoubtedly more complex than in vitro, achieving an initial burst of hydroxycamptothecin followed by sustained release appears to play an important role in its efficacy observed here.

The safe and effective local delivery of hydroxycamptothecin from biodegradable polymers avoids the unacceptable toxicity associated with systemic delivery and provides an important option, as topoisomerase inhibitors are further considered for the clinical management of malignant glioma.

\section{References}

[1] Menei, P. (2005) Local and Sustained Delivery of 5-Fluorouracil from Biodegradable Microspheres for the Radiosensitization of Malignant Glioma: A Randomized Phase II Trial. Neurosurgery, 56, 242-248. http://dx.doi.org/10.1227/01.NEU.0000144982.82068.A2

[2] Preface (2006) Local Controlled Drug Delivery to the Brain. International Journal of Pharmaceutics, 314, 99-100.

[3] Storm, P.B., Moriarity, J.L. and Tyler, B. (2002) Polymer Delivery of Camptothecin against 9L Gliosarcoma: Release, Distribution and Efficacy. Neuro-Oncology, 56, 209-217.

[4] Zhang, Z.W., Patchett, S.E. and Farthing, M.J. (2000) Topoisomerase I Inhibitor (Camptothecin)-Induced Apoptosis in Human Gastric Cancer Cells and the Role of Wild-Type p53 in the Enhancement of Its Cytotoxicity. Anticancer Drugs, 11, 57-64. http://dx.doi.org/10.1097/00001813-200010000-00013

[5] Zhang, R., Li, Y., Cai, Q., Liu, T., Sun, H. and Chambless, B. (1998) Preclinical Pharmacology of the Natural Product Anticancer Agent 10-Hydroxycamptothecin, an Inhibitor of Topoisomerase I. Cancer Chemotherapy and Pharmacology, 41, 257-267. http://dx.doi.org/10.1007/s002800050738

[6] Fassberg, J., Stella, V.J., Wang, S.L., Lin, S.Y., Hsieh, T.F. and Chan, S.A. (2007) Thermal Behavior and Thermal Decarboxylation of 10-Hydroxycamptothecin in the Solid State. Journal of Pharmaceutical and Biomedical Analysis, 43, 457-463. http://dx.doi.org/10.1016/j.jpba.2006.07.023

[7] Takahashi, M., Onishi, H. and Machida, Y. (2004) Development of Implant Tablet for a Week-Long Sustained Release. Journal of Controlled Release, 100, 63-74. http://dx.doi.org/10.1016/j.jconrel.2004.07.031

[8] Gusea, C. and Koennings, S. (2006) Biocompatibility and Erosion Behavior of Implants Made of Triglycerides and Blends with Cholesterol and Phospholipids. International Journal of Pharmaceutics, 314, 153-160. http://dx.doi.org/10.1016/j.ijpharm.2005.12.050

[9] Gusea, C. and Koennings, S. (2006) Programmable Implants—From Pulsatile to Controlled Release. International Journal of Pharmaceutics, 314, 161-169. http://dx.doi.org/10.1016/j.ijpharm.2005.12.051

[10] Dang, W.B., Daviau, T. and Ying, P. (1996) Effects of GLIADEL ${ }^{\circledR}$ Wafer Initial Molecular Weight on the Erosion of Wafer and Release of BCNU. Journal of Controlled Release, 42, 83-92. http://dx.doi.org/10.1016/0168-3659(96)01371-5

[11] Ma, M.-F., Yu, T., Dai, S.-J., Wang, Y. and Yan, X.-F. (2002) Determination of Contents of 10-Hydroxycamptothecin in Camptotheca Acuminata by High-Performance Liquid Chromatogram. Journal of Forestry Research, 13, $144-146$. http://dx.doi.org/10.1007/BF02857241

[12] Naraharisetti, P.K., Ong, B.Y.S. and Xie, J.W. (2007) In Vivo Performance of Implantable Biodegradable Preparations Delivering Paclitaxel and Etanidazole for the Treatment of Glioma. Biomaterials, 28, 886-894. http://dx.doi.org/10.1016/j.biomaterials.2006.09.044

[13] Ulmer, S., Reeh, M. and Krause, J. (2008) Dynamic Contrast-Enhanced Susceptibility-Weighted Perfusion MRI (DSC-MRI) in a Glioma Model of the Rat Brain Using a Conventional Receive-Only Surface Coil with a Inner Diameter of $47 \mathrm{~mm}$ at a Clinical $1.5 \mathrm{~T}$ Scanner. Journal of Neuroscence Methods, 172, 168-172. http://dx.doi.org/10.1016/j.jneumeth.2008.04.022

[14] Fu, Y.-R., Yi, Z.-J. and Yan, Y.-R. (2006) Hydroxycamptothecin-Induced Apoptosis in Hepatoma SMMC-7721 Cells and the Role of Mitochondrial Pathway. Mitochondrion, 6, 211-217. http://dx.doi.org/10.1016/j.mito.2006.07.005

[15] Zhang, L.Y., Yang, M. and Wang, Q. (2007) 10-Hydroxycamptothecin Loaded Nanoparticles: Preparation and Antitumor Activity in Mice. Journal of Controlled Release, 119, 153-162. http://dx.doi.org/10.1016/j.jconrel.2007.02.013

[16] Vochelle, D. (2004) The Use of Poly-L-Lactic Acid in the Management of Soft-Tissue Augmentation: A Five-Year Experience. Seminars in Cutaneous Medicine and Surgery, 23, 223-226. http://dx.doi.org/10.1016/j.sder.2004.09.001

[17] Freitas, M.N. and Marchetti, J.M. (2005) Nimesulide PLA Microspheres as a Potential Sustained Release System for the Treatment of Inflammatory Diseases. International Journal of Pharmaceutics, 295, 201-211. http://dx.doi.org/10.1016/j.ijpharm.2005.03.003

[18] Guo, X.D., Zhang, L.J. and Qian, Y. (2007) Effect of Composition on the Formation of Poly(dl-lactide) Microspheres for Drug Delivery Systems: Mesoscale simulations. Chemical Engineering Journal, 131, 195-201. http://dx.doi.org/10.1016/j.cej.2007.01.013 
[19] Olivi, A., Ewend, M.G., Utsuki, T., Tyler, B., Domb, A.J., Brat, D.J. and Brem, H. (1996) Interstitial Delivery of Carbopla Tin via Biodegradable Polymers Is Effective against Experimental Glioma in the Rat. Cancer Chemotherapy and Pharmacology, 39, 90-96. http://dx.doi.org/10.1007/s002800050542 
Scientific Research Publishing (SCIRP) is one of the largest Open Access journal publishers. It is currently publishing more than 200 open access, online, peer-reviewed journals covering a wide range of academic disciplines. SCIRP serves the worldwide academic communities and contributes to the progress and application of science with its publication.

Other selected journals from SCIRP are listed as below. Submit your manuscript to us via either submit@scirp.org or Online Submission Portal.
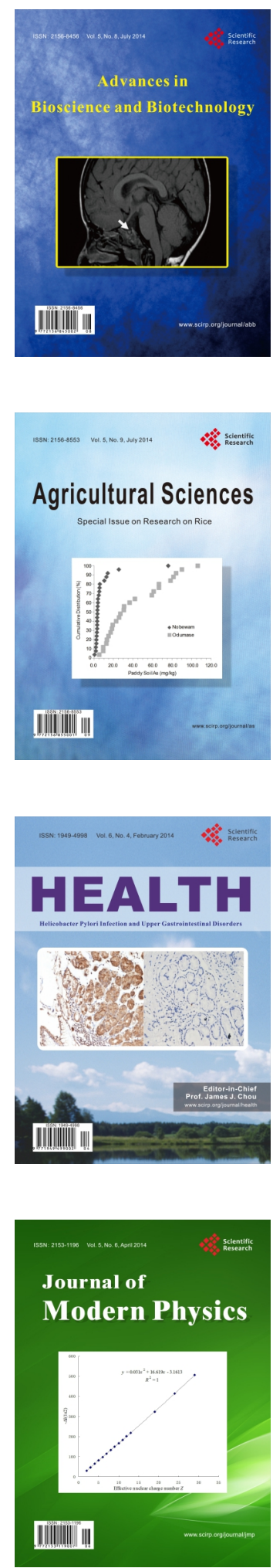
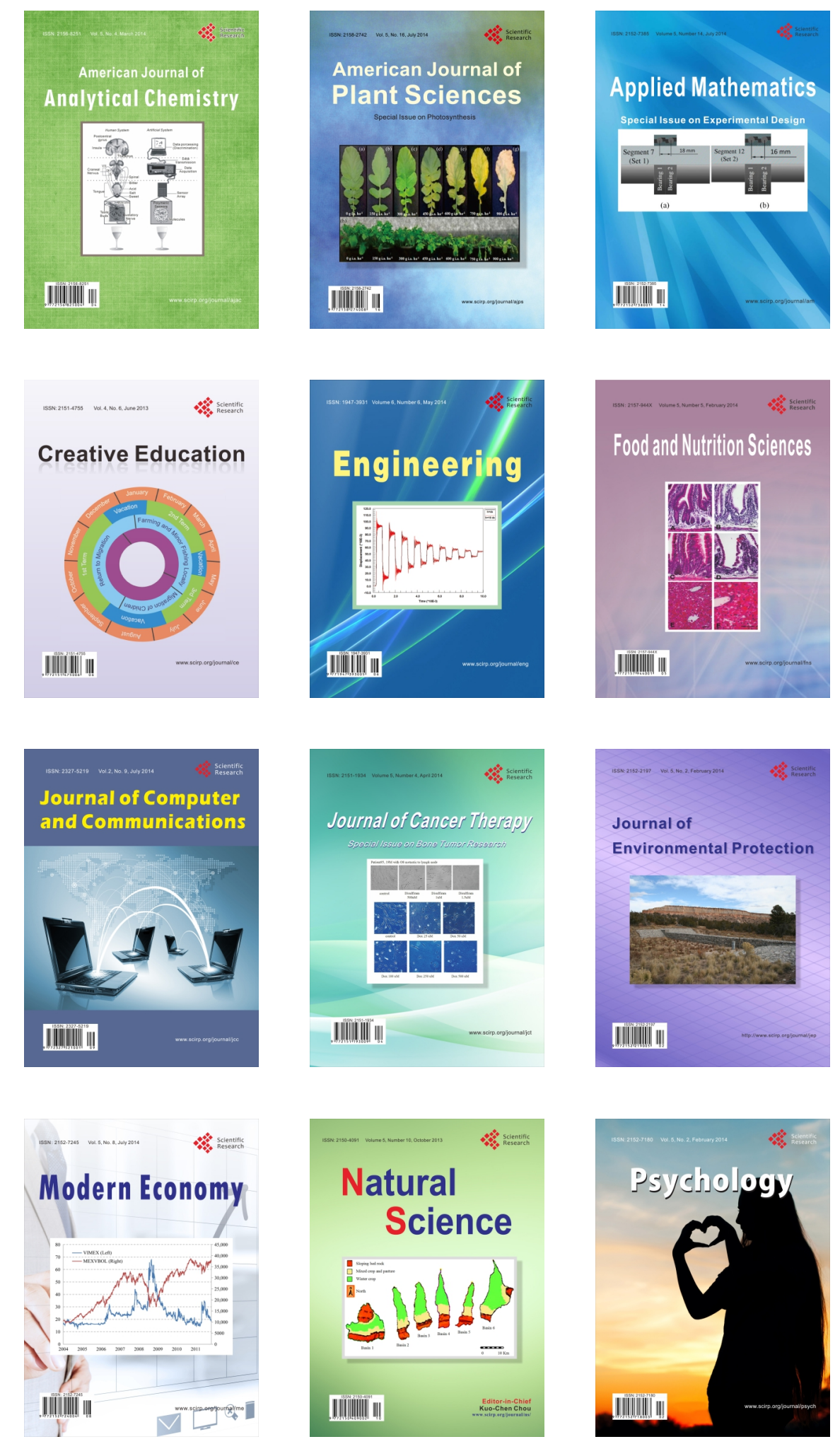